\title{
Necesidades de capacitación profesional en educación para adultos del personal docente y administrativo de colegios académicos nocturnos
}

Erick Víquez Monge

Auxiliar administrativo y profesional técnico docente, Ministerio de Educación Pública, Costa Rica.

erick.viquez.monge@mep.go.cr

Recibido: 12 de agosto del 2018 Corregido: 05 de setiembre del 2018 Aceptado: 17 de setiembre del 2018

\section{Resumen}

El propósito del artículo es presentar los resultados de un estudio efectuado en el Colegio Nocturno La Unión (CNLU) que buscó detectar las deficiencias que a nivel de formación andragógica muestra el personal.

A partir de lo anterior se esbozó una propuesta de capacitación dirigida al personal del centro de estudios, la cual promueva el desarrollo profesional continuo mediante la adquisición de habilidades profesionales en temáticas andragógicas.

La información recolectada fue analizada y contrastada metodológicamente a través de un diagnóstico inicial, basado en un enfoque mixto. El análisis final se efectuó mediante la triangulación concurrente que determina la convergencia de la información final y las conclusiones generales.

El estudio evidencia la necesidad de capacitación tanto para el personal activo como en profesionales actualmente en formación universitaria, según los resultados obtenidos el abordaje utilizado para atender a los estudiantes adultos no es el más adecuado, se aboga por un proceso educativo más diferenciado.

Finalmente, se logran definir características personales y profesionales de un administrador educativo de instituciones para adultos, en donde la administración educativa estratégica se convierte en un importante instrumento de cambio, a partir del cual se puede mejorar la gestión institucional y los procesos educativos de los discentes adultos.

Palabras clave: Gestión Educativa Estratégica, Andragogía, Educación de Adultos, Educación de Jóvenes y Adultos, Capacitación, Formación Profesional

\footnotetext{
Abstract

The purpose of this article is to present the results of a research applied at Colegio Nocturno La Unión (CNLU) that pretended to identify the lacks in the andragogy teaching processes in this institution.

Based on the previous information, a draft of a proposal for a professional training session was prepared; this idea wants to promote the continuous professional development through the acquisition of effective skills in andragogy topics.

The collected information was analyzed and contrasted methodologically by using an initial diagnose, based on a mixed approach. The final analysis was performed through a concurrent triangulation, which determined the convergence of the final information and the general conclusions.
} 
The research shows the need of having appropriate training, not only for the current personnel, but also for the future professionals who are studying in the universities. According to the results, the approach used to teach adult students is not the most accurate; for that reason, it is recommended to have a more personalized teaching process based on the students' needs.

Finally, there are defined, personal and professional characteristics of a school administrator of adult institutions, where the educative administration becomes a strategically important tool for a change in the andragogy process, with the purpose to improve the institutional management and the educative development of adult learners.

Key words: Strategic Educative Management, Andragogy, Adults' Education, Youth and Adult Education, Professional Development, Professional Training.

\section{INTRODUCCIÓN}

Actualmente, tanto a nivel nacional como latinoamericano la realidad social exige destinar mayores recursos a la educación; es indispensable trabajar en políticas educativas que permitan mejorar la calidad de la educación, la gestión de los centros educativos, reducir el bajo rendimiento académico y erradicar la falta de equidad existente.

En este sentido, el Ministerio de Educación de la Nación de Argentina (2015), menciona que es necesario volver a pensar las modalidades organizativas y directivas de los sistemas educacionales. Los administradores educativos, quienes tienen a cargo su gestión, enfrentan una tarea compleja como nunca antes, la cual requiere un alto nivel de competencia profesional, por lo tanto, las instituciones deben adecuarse a la realidad inminente.

Dentro de dicha complejidad se ubican los colegios nocturnos, los cuales son sustancialmente diferentes a cualquier otro centro educativo de la misma modalidad con un horario diurno, principalmente por el tipo de población estudiantil, en su mayoría personas adultas.

Cabe destacar cómo a nivel nacional tanto los docentes como los administradores educativos son formados profesionalmente desde los principios de la pedagogía, no obstante, en este artículo se evidencia la necesidad de capacitación profesional en educación de personas jóvenes y adultas específicamente en andragogía, la cual según Yturralde (2016), es la disciplina que se ocupa de la educación y el aprendizaje del adulto hasta su madurez.

Es relevante entonces tomar en consideración que en la actualidad se vive una época de muchas transformaciones tanto en el área tecnológica como social, las cuales surten efectos positivos o negativos en la educación.

El presente artículo enfatiza en la necesidad de que los centros educativos sean administrados con liderazgo y capacidad para gestionar eficientemente los aspectos administrativos, organizativos, curriculares y comunitarios; sin embargo, es claro que son necesarios cambios que permitan adaptar el proceso educativo a las necesidades de los discentes y al entorno donde están inmersos.

De acuerdo con los aspectos mencionados, se deben buscar mecanismos que propicien el cambio, una de las principales problemáticas de las instituciones educativas académicas nocturnas se refiere a los propios administradores educativos, los cuales no han desarrollado a lo largo del tiempo estrategias que permitan adaptar la gestión y el proceso educativo a la realidad.

Relacionado a lo anterior, según la Comisión Nacional de Rectores (CONARE) (2015), en su informe sobre el Estado de la Educación, indica que los colegios académicos nocturnos se caracterizan por su bajo 
rendimiento académico y altos índices de deserción estudiantil, por lo tanto, es una situación que debe atenderse con urgencia.

Según lo expuesto, es evidente que deben darse cambios, ante esta necesidad se aboga por la aplicación de una administración basada en la gestión educativa estratégica que tome en cuenta los principios andragógicos y que propicie procesos de capacitación profesional.

En este artículo se exponen los resultados de la investigación que se llevó a cabo en el Colegio Nocturno La Unión en adelante conocido como "CNLU" cuyos principales objetivos se enfocaron en diagnosticar cuáles son las necesidades de formación profesional del personal docente y técnico docente con respecto al proceso educativo de los discentes adultos, basándose para tal fin en los principios andragógicos.

\section{REFERENTES TEÓRICOS}

La educación moldea a todos los seres humanos, nuestra madre brindó aprendizaje a través de sus primeros cuidados posteriormente la interacción con familiares, amigos, compañeros en escuelas y colegios son experiencias educativas que configuran a lo largo del tiempo el modo de ser.

Relacionado a lo anterior, Freire (1972) brinda una definición de educación muy interesante, la cual es muy atinada a los fines del presente artículo, para él se define desde un enfoque más humanista al mencionar que es un arma vital para la liberación del pueblo y la transformación de la sociedad, es un constante vivir experiencias mutuas entre el educador y el educando, es el arma de lucha contra el atraso y la pobreza.

Tener una visión de educación más humanista es sumamente importante al hablar de una modalidad educativa para adultos, dadas las características que presentan los discentes, donde en muchos casos son vulnerables, con dificultades familiares, laborales y personales, que hacen del proceso educativo y administrativo todo un reto.

Ahora bien, en este punto es importante definir también qué es educación de adultos, la UNESCO (1985) define educación de adultos de la siguiente manera:

La designación de la totalidad de los procesos organizados de educación, sea cual sea el contenido, el nivel o el método, sean formales o no formales, ya sea que prolonguen o reemplacen la educación inicial dispensada en las escuelas, universidades y forma de aprendizaje profesional, gracias a las cuales, las personas consideradas como adultas por la sociedad a la que pertenecen, desarrollan sus aptitudes, enriquecen sus conocimientos, mejoran sus competencias técnicas o profesionales y hacen evolucionar sus aptitudes o su comportamiento en la doble perspectiva de un enriquecimiento integral del hombre y una participación en el desarrollo socioeconómico e independiente. (p. 9)

Según lo anterior, este proceso educativo debe ser diferenciado, si la educación de niños y adolescentes se basa en los principios de la pedagogía, es importante reflexionar entonces que para educar discentes adultos lo correspondiente es tomar en consideración los principios de la andragogía.

Al respecto, Sanz (2002) explica que la pedagogía es aplicada en niños y adolescentes utilizando métodos didácticos para el aprendizaje por lo cual el estudiante es más un receptor que un participante y es influenciado por factores externos como la motivación extrínseca.

Por otra parte, la andragogía para Knowles (2005) es la disciplina que se ocupa de la educación y el aprendizaje del adulto, considera que los estudiantes necesitan ser participantes activos en su propio 
aprendizaje, ellos aprenden de manera diferente a los niños y los facilitadores del aprendizaje deberían usar un proceso diferente.

Recordemos también que la educación no es solo cuestión de niños y jóvenes es un proceso que debe actuar sobre las personas durante toda su vida, la naturaleza del hombre demuestra que puede aprender a lo largo de su existencia sin importar su edad.

Ahora bien, dentro de la dinámica de las instituciones educativas un aspecto fundamental para su correcto funcionamiento corresponde a su administración, en este sentido es importante definir términos como la gestión educativa estratégica, área encargada propiamente de la administración institucional para que todos los componentes educativos se integren de manera armoniosa.

El Ministerio de Educación de la Nación de Argentina (2015) define la gestión educativa estratégica de la siguiente manera:

La gestión educativa estratégica es vista como un conjunto de procesos teórico-prácticos integrados horizontal y verticalmente dentro del sistema educativo, para cumplir los mandatos sociales. Es un saber de síntesis capaz de ligar conocimiento y acción, ética y eficacia, política y administración en procesos que tienden al mejoramiento continuo de las prácticas educativas; a la exploración y explotación de todas las posibilidades; y a la innovación permanente como proceso sistemático. (p. 16)

Por lo tanto, los procesos de gestión implican aquellas acciones desplegadas por quienes dirigen la educación, como un solo eje que toma en consideración conocimiento y acción, ética y eficacia, política y administración, con el fin de mejorar permanentemente las prácticas educativas.

Una de las alternativas, dentro de los procesos de mejora del proceso educativo por parte del administrador educativo, es brindar la posibilidad y los espacios para que el personal acceda a procesos de capacitación y actualización profesional.

Uno de los grandes referentes en la temática de capacitación es Chiavenato (2009), quien define capacitación de la siguiente manera:

La capacitación es el proceso de modificar, sistemáticamente, el comportamiento de los empleados con el propósito de que alcancen los objetivos de la organización. La capacitación se relaciona con las habilidades y las capacidades que exige actualmente el puesto. Su orientación pretende ayudar a los empleados a utilizar sus principales habilidades y capacidades para poder alcanzar el éxito. (p. 371)

Por lo tanto, a través de una eficiente gestión estratégica que implemente procesos de capacitación es posible brindar la oportunidad de que el personal docente y técnico docente de la institución adquiera las herramientas necesarias para brindar una atención más acorde con la población estudiantil adulta que atiende.

La formación continua o permanente se erige en un extraordinario medio profesionalizador; según Imbernón (2007), la formación se encuentra vinculada directamente al desarrollo profesional docente, entendido como una actitud de constante aprendizaje, así la formación y el desarrollo profesional forman un requisito indispensable y necesario para desempeñar la profesión educativa. 


\section{MATERIALES Y MÉTODOS}

La metodología empleada consistió en el desarrollo de un diagnóstico institucional con el fin de determinar las necesidades de formación del personal del CNLU, enfocándose para tal fin en los principios andragógicos, puesto que se realizó en un centro educativo con discentes adultos. El principal objetivo fue implementar acciones de formación continua que desde la figura del administrador educativo propiciaran la adquisición de habilidades profesionales.

Con respecto al personal que fue objeto de estudio, se consideraron 39 docentes (17 hombres y $22 \mathrm{mu}$ jeres), 7 miembros del personal técnico docente y administrativo (2 hombres y 5 mujeres), que incluía a la administración (director, subdirector, auxiliares administrativos) y al departamento de orientación y bibliotecología.

Antes de indicar algunos detalles clave de la metodología empleada en la investigación es importante aclarar que el proyecto se desarrolló desde un enfoque mixto, cualitativo y cuantitativo, Chen (2006, citado por Hernández, Fernández y Baptista, 2010) agrega, "[...] los métodos de investigación mixta son la integración sistémica de los métodos cuantitativo y cualitativo en un solo estudio con el fin de obtener una "fotografía" más completa del fenómeno" (p. 546).

Metodológicamente, todo el proceso de investigación se dividió en tres fases principales, una primera etapa se desarrolló por medio de una indagación teórica sobre la andragogía, gestión educativa, colegios nocturnos y procesos de capacitación.

La segunda etapa planteó el establecimiento de las categorías y variables de la investigación acordes con los objetivos planteados, se incluyó también la metodología empleada en la validación de los instrumentos para recolectar la información dentro de los cuales se encontraba la encuesta escrita llamada guía de cuestionario y la aplicación de entrevistas estructuradas en profundidad al personal docente administrativo, por medio de las cuales se logró analizar cualidades, contextos y fenómenos sociales desde un paradigma naturalista. Los resultados fueron analizados estadísticamente con el fin de obtener generalizaciones a través de un paradigma positivista.

Para la tercera etapa, dentro del enfoque mixto de la pesquisa efectuada se utilizó el diseño de la triangulación concurrente, en la cual a partir de las tres fuentes de información mencionadas anteriormente: las entrevistas, los cuestionarios y la que nos facilita el marco teórico referencial, se determinó la convergencia de las mismas en una gráfica que permitió visualizar claramente los resultados finales, e identificar así por último la incidencia de los aspectos previos en la gestión y proceso educativo desarrollado en el CNLU.

Después de un extenso análisis de la información generada en el marco teórico y la consulta con especialistas en la materia fue posible definir las categorías asociadas con los datos cualitativos y las variables con los datos cuantitativos, las cuales se presentan en la tabla 1. 
TABLA 1

Operacionalización de variables y definición de categorías

\begin{tabular}{|c|c|c|}
\hline Variables y categorías & Objetivo de su utilización & Referente teórico \\
\hline $\begin{array}{l}\text { Necesidades de formación } \\
\text { continua del personal } \\
\text { docente y técnico docente }\end{array}$ & $\begin{array}{l}\text { Diagnosticar las necesidades } \\
\text { de formación continua } \\
\text { del personal docente } \\
\text { y técnico docente que } \\
\text { afectan el desarrollo de } \\
\text { la gestión institucional } \\
\text { desde su dimensión } \\
\text { andragógica-curricular. }\end{array}$ & $\begin{array}{l}\text { Según Camargo (2007), "Las necesidades de formación continua } \\
\text { son aquellas formuladas por los docentes y que se encuentran } \\
\text { ancladas en su práctica pedagógica y en el trabajo que realizan } \\
\text { a nivel institucional" (p. 6). }\end{array}$ \\
\hline $\begin{array}{l}\text { Características profesionales } \\
\text { del director educativo para } \\
\text { el ejercicio de la gestión } \\
\text { educativa estratégica }\end{array}$ & $\begin{array}{l}\text { Determinar las } \\
\text { características profesionales } \\
\text { del director educativo para } \\
\text { el ejercicio de la gestión } \\
\text { educativa estratégica bajo } \\
\text { una modalidad educativa } \\
\text { andragógica. }\end{array}$ & $\begin{array}{l}\text { Según Navío (2005), las características profesionales son } \\
\text { un conjunto de elementos combinados (conocimientos, } \\
\text { habilidades, actitudes, saberes, etc.), que se integran } \\
\text { atendiendo a una serie de atributos personales (capacidades, } \\
\text { motivos, rasgos de la personalidad, aptitudes, etc.), tomando } \\
\text { como referencia las experiencias personales y profesionales } \\
\text { que se manifiestan mediante determinados comportamientos o } \\
\text { conductas en el contexto de trabajo. }\end{array}$ \\
\hline $\begin{array}{l}\text { Acciones de formación } \\
\text { continua que estimulen } \\
\text { la adquisición de las } \\
\text { habilidades profesionales }\end{array}$ & $\begin{array}{l}\text { Diseñar desde la gestión } \\
\text { educativa estratégica } \\
\text { acciones de formación } \\
\text { continua que estimulen } \\
\text { la adquisición de las } \\
\text { habilidades profesionales. }\end{array}$ & $\begin{array}{l}\text { Según Galiano (2006), la formación continua está destinada a } \\
\text { los empleados, con la finalidad de dotarles de los conocimientos } \\
\text { y habilidades requeridos, brindándoles la posibilidad de } \\
\text { desempeñar su trabajo con mayor eficacia y de mejorar sus } \\
\text { condiciones laborales, facilitándoles su especialización. }\end{array}$ \\
\hline
\end{tabular}

Fuente: Elaboración propia basada en Víquez (2017).

De acuerdo con la definición de las variables descritas en la tabla 1 se procedió a elaborar el instrumento guía de cuestionario que se aplicó a los docentes; por otra parte, con respecto a las categorías, con base en ellas se desarrolla la guía de entrevista, la cual forma parte de las técnicas de entrevista utilizadas para recolectar información de tipo cualitativo que fueron aplicadas al personal técnico y administrativo docente.

El análisis final se realizó utilizando la técnica de triangulación, a partir de los resultados obtenidos de los datos cuantitativos mediante el análisis estadístico en el programa Microsoft Excel (promedios, frecuencias absolutas y porcentuales).

Se efectuó posteriormente un análisis de los datos cualitativos basados en lo mencionado por Hurtado (2010) "[...] una vez aplicados los instrumentos y finalizada la tarea de recolección de información, el investigador debe organizarla, procesarla y desarrollar un tipo de análisis que le permita llegar a una conclusión con base en los objetivos planteados al principio [...]" (p. 325).

\section{DISCUSIÓN DE RESULTADOS}

El apartado presenta los resultados más importantes de la investigación, basados en algunas de las variables y categorías del estudio, según la temática planteada en el presente artículo. Estos y su discusión se dividen para cada apartado en una primera parte con los resultados cuantitativos y posteriormente con los cualitativos, para así tener una visión más integral del estudio efectuado. 


\section{Necesidades de formación continua del personal}

En este apartado se pretende indagar sobre si existe necesidad de formación continua en el personal del CNLU. De acuerdo con los resultados obtenidos a partir del cuestionario aplicado a los docentes, se puede observar en la tabla 2 como todas las variables fueron significativas en la investigación, pues el promedio de las respuestas positivas dadas a cada una de ellas es de 95,4\% (33 docentes en promedio) muy superior al promedio de respuestas negativas que corresponde a 4,6\% ( 2 docentes en promedio).

Según los resultados mostrados en la tabla 2, para los docentes encuestados existe una gran necesidad de formación continua en temáticas como el proceso de enseñanza y aprendizaje y contar con un mayor conocimiento sobre las características de los estudiantes adultos; además, consideran necesario recibir capacitación en métodos educativos para trabajar con discentes adultos y sobre cómo tener un control y manejo de aula con este tipo de población estudiantil.

Acorde con lo antes expuesto queda en evidencia cierto grado de desconocimiento a nivel docente sobre el correcto abordaje educativo de estudiantes adultos en las aulas, no obstante, se debe tener en cuenta que los docentes son formados profesionalmente en los principios de la pedagogía.

TABLA 2

Resumen de las respuestas dadas por los docentes con respecto a las necesidades de formación continua del personal

\begin{tabular}{|c|c|c|c|c|}
\hline \multirow[b]{2}{*}{ Variables } & \multicolumn{2}{|c|}{$\begin{array}{l}\text { Respuestas negativas: } \\
\text {-Muy en desacuerdo } \\
\text {-En desacuerdo } \\
\text {-Nide acuerdo ni en desacuerdo }\end{array}$} & \multicolumn{2}{|c|}{$\begin{array}{l}\text { Respuestas positivas: } \\
\text {-Muy de acuerdo } \\
\text {-De acuerdo }\end{array}$} \\
\hline & $\begin{array}{c}\text { Frecuencia } \\
\text { absoluta }\end{array}$ & $\begin{array}{l}\text { Frecuencia } \\
\text { relativa }\end{array}$ & $\begin{array}{c}\text { Frecuencia } \\
\text { absoluta }\end{array}$ & $\begin{array}{c}\text { Frecuencia } \\
\text { relativa }\end{array}$ \\
\hline $\begin{array}{l}\text { Pregunta 5: ¿Considera necesario que se le brinde una capa- } \\
\text { citación sobre cómo desarrollar el proceso de enseñanza y } \\
\text { aprendizaje en el aula con estudiantes adultos? }\end{array}$ & 2 & $6 \%$ & 33 & $94 \%$ \\
\hline $\begin{array}{l}\text { Pregunta 6: ¿Es necesario recibir algún tipo de capacitación } \\
\text { para trabajar en un colegio nocturno a partir de las caracte- } \\
\text { rísticas de los estudiantes que se atienden? }\end{array}$ & 0 & $0 \%$ & 35 & $100 \%$ \\
\hline $\begin{array}{l}\text { Pregunta 7: ¿Basándose en el perfil profesional con el cual } \\
\text { usted se graduó de la universidad, considera usted que son } \\
\text { necesarias capacitaciones para trabajar con estudiantes } \\
\text { adultos en el colegio nocturno? }\end{array}$ & 1 & $3 \%$ & 34 & $97 \%$ \\
\hline $\begin{array}{l}\text { Pregunta 8: ¿Son necesarias capacitaciones que le permitan } \\
\text { desarrollar metodologías educativas que se ajusten a la po- } \\
\text { blación estudiantil que se atiende en el colegio nocturno? }\end{array}$ & 0 & $0 \%$ & 35 & $100 \%$ \\
\hline $\begin{array}{l}\text { Pregunta 9: ¿Considera necesarias capacitaciones sobre el } \\
\text { control y manejo de los estudiantes adultos en el aula en el } \\
\text { colegio nocturno? }\end{array}$ & 5 & $14 \%$ & 30 & $86 \%$ \\
\hline Promedio & 1,6 & $4,6 \%$ & 33,4 & $95,4 \%$ \\
\hline
\end{tabular}

Fuente: Víquez (2017, p. 160). 
Se puede visualizar en la tabla 2 que los docentes manifiestan que existe un vacío importante a nivel de formación profesional para trabajar con adultos, aspecto que sobresale a partir de las respuestas dadas en la pregunta 7 del cuestionario.

Con respecto a la categoría "necesidades de formación continua" se analizaron algunas subcategorías como: las metodologías de enseñanza en el aula, el currículo educativo, la evaluación de los estudiantes y el perfil docente.

En la entrevista efectuada a la subdirectora, ella menciona con respecto de las metodologías de enseñanza lo siguiente:

[...] el abordaje a nivel de aula y a nivel administrativo es completamente distinto... (Además expresa) ...son poblaciones muy heterogéneas y definitivamente las necesidades son completamente distintas [...] (Sánchez, 2017).

Lo anterior enfocado en que los docentes en un colegio nocturno no pueden utilizar las mismas metodologías pedagógicas empleadas en un colegio diurno porque es completamente distinto, pues al ser poblaciones estudiantiles diferentes las necesidades educativas son otras.

Con base en estas declaraciones y los resultados obtenidos es válida la siguiente pregunta ¿por qué se utilizan programas educativos diseñados bajo los principios de la pedagogía en instituciones educativas para adultos?, la realidad muestra que es el propio personal docente y administrativo quien debe hacer ajustes para lograr éxito en el proceso educativo.

Otro punto importante mencionado por los entrevistados fue el perfil profesional docente, ya que consideran que existen necesidades de formación andragógica, por ejemplo, la subdirectora opina lo siguiente:

[...] está enfocada en la niñez y la adolescencia prácticamente, no hay todavía o al menos a lo que yo sé, algún curso especializado en andragogía [...] (Sánchez, 2017).

\section{Características profesionales del director (a) de un colegio nocturno}

¿Un director de un colegio nocturno debe ser un profesional capacitado en educación de adultos?, ¿debe poseer además algunas características específicas? Conforme con los resultados encontrados en los cuestionarios aplicados a los docentes se comprueba la importancia de las características profesionales de un administrador educativo para una modalidad de educación de adultos.

Las respuestas a las interrogantes anteriores se obtuvieron al preguntarles a los docentes si es importante que un director de una institución educativa académica para adultos sea un profesional propiciador del trabajo en equipo, lidere acciones que mejoren el proceso de enseñanza y aprendizaje, establezca un contacto con la comunidad y sus necesidades, sea un líder capaz de resolver de manera asertiva los conflictos, tenga un buen dominio de la gestión educativa estratégica, sea un profesional con formación en andragogía y posea algunas características personales especiales.

En la tabla 3, se observan los resultados obtenidos a nivel cuantitativo, todas las variables son significativas porque el promedio de las respuestas positivas dadas a cada una de ellas es de un $96,6 \%$ ( 34 docentes en promedio). 
TABLA 3

Resumen de respuestas dadas por los docentes con respecto a las características profesionales del director (a)

\begin{tabular}{|c|c|c|c|c|}
\hline \multirow[b]{2}{*}{ Variables } & \multicolumn{2}{|c|}{$\begin{array}{l}\text { Respuestas negativas: } \\
\text {-Muy en desacuerdo } \\
\text {-En desacuerdo } \\
\text {-Ni de acuerdo ni en desacuerdo }\end{array}$} & \multicolumn{2}{|c|}{$\begin{array}{l}\text { Respuestas positivas: } \\
\text {-Muy de acuerdo } \\
\text {-De acuerdo }\end{array}$} \\
\hline & $\begin{array}{c}\text { Frecuencia } \\
\text { absoluta }\end{array}$ & $\begin{array}{c}\text { Frecuencia } \\
\text { relativa }\end{array}$ & $\begin{array}{c}\text { Frecuencia } \\
\text { absoluta }\end{array}$ & $\begin{array}{c}\text { Frecuencia } \\
\text { relativa }\end{array}$ \\
\hline $\begin{array}{l}\text { Pregunta 10: ¿Considera necesario que un director de un } \\
\text { colegio nocturno debe propiciar el trabajo en equipo de todo } \\
\text { el personal? }\end{array}$ & 1 & $3 \%$ & 34 & $97 \%$ \\
\hline $\begin{array}{l}\text { Pregunta 11: ¿Considera necesario que un director de un cole- } \\
\text { gio nocturno debe propiciar acciones en pro de la mejora del } \\
\text { proceso de enseñanza-aprendizaje de los estudiantes? }\end{array}$ & 1 & $3 \%$ & 34 & $97 \%$ \\
\hline $\begin{array}{l}\text { Pregunta 12: ¿Considera que un director de un colegio noc- } \\
\text { turno debe propiciar acciones que involucren a la comunidad } \\
\text { donde se encuentra la institución educativa? }\end{array}$ & 4 & $11 \%$ & 31 & $89 \%$ \\
\hline $\begin{array}{l}\text { Pregunta 13: ¿Considera que un director de un colegio noc- } \\
\text { turno debe propiciar acciones para una buena resolución de } \\
\text { conflictos? }\end{array}$ & 0 & $0 \%$ & 35 & $100 \%$ \\
\hline $\begin{array}{l}\text { Pregunta } 14: \text { ¿Considera que un director de un colegio noctur- } \\
\text { no debe ejecutar acciones oportunas de planificación y gestión } \\
\text { institucionales? }\end{array}$ & 0 & $0 \%$ & 35 & $100 \%$ \\
\hline Promedio & 1,2 & $3,4 \%$ & 33,8 & $96,6 \%$ \\
\hline
\end{tabular}

Fuente: Víquez (2017, p. 164)

Según los resultados obtenidos en la tabla 3, surge la siguiente pregunta: ¿cómo un director de una institución educativa para adultos es capaz de desarrollar un proceso de gestión que tome en cuenta lo mencionado anteriormente?, uno de los aspectos de mayor énfasis corresponde a que tanto docentes como directores deben recibir una formación profesional más integral que los prepare no solamente para enfrentarse a ambientes educativos de índole pedagógico, asimismo estén preparados para desenvolverse profesionalmente en cada una de las opciones profesionales del sistema educativo costarricense.

En el caso específico de este estudio, a partir de los resultados cuantitativos obtenidos se evidencia la necesidad de que un director de una institución educativa de adultos sea formado tomando en consideración los principios de la gestión educativa estratégica fundamentados desde una perspectiva andragógica.

Junto a lo anterior, los resultados cualitativos respaldan los cuantitativos, con respecto al perfil profesional de un director para un colegio académico nocturno. En la figura 1, se evidencian algunos de los principales aspectos mencionados en las entrevistas con respecto a las características profesionales de un director de un colegio académico nocturno.

A partir de los resultados presentados en la figura 1, un director de un colegio académico nocturno debe ser un profesional con características personales marcadas, quien debe tener tanto sensibilidad como temple para conquistar y valorar a los estudiantes que asisten a este tipo de instituciones porque son muy vulnerables, además se considera indispensable que el director sea conciliador, así como un buen 
líder que sepa escuchar tanto a docentes como a estudiantes.

Al respecto la auxiliar administrativa es enfática al decir que el director de un centro educativo para adultos debe ser formado profesionalmente en educación andragógica:

[...] un director debe ser formado en andragogía [...] (Duarte, 2017).

Por otra parte, la subdirectora mencionó que el director debe ser una persona especial, al decir:

[...] una de las características principales es un carisma especial, con el trato a nivel de estudiantes y con el trato del personal docente [...] (Sánchez, 2017).

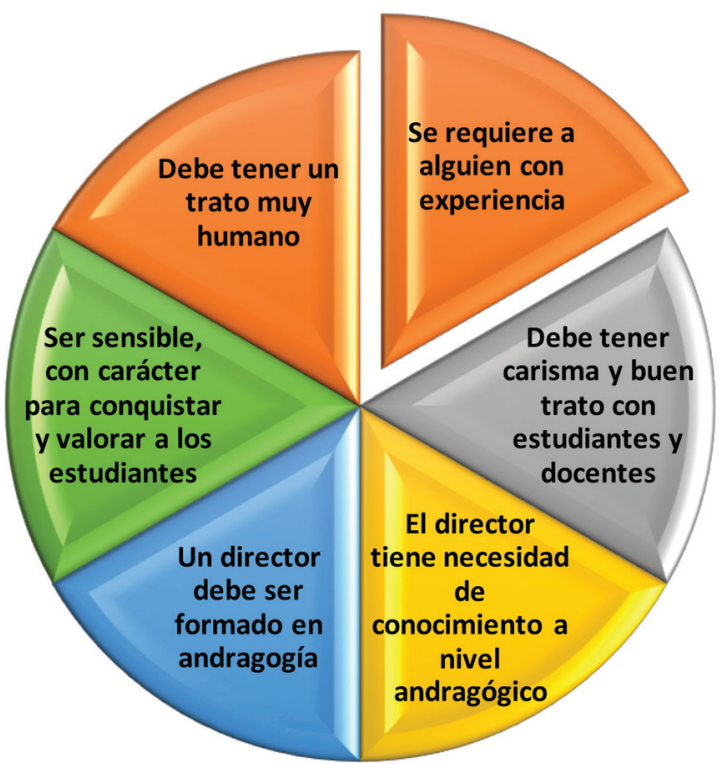

Figura 1. Características profesionales del director educativo para el ejercicio de la gestión educativa estratégica. Fuente: Elaboración propia basada en Víquez (2017).

\section{Acciones de formación continua que estimulen la adquisición de habilidades profesionales}

En este apartado se les preguntó a los docentes sobre las acciones que a nivel de dirección se ejecutan para solventar de alguna manera las necesidades de formación del personal.

Desde la perspectiva de la gestión educativa estratégica, se debe pensar en las acciones derivadas de la dirección con el fin de brindar la posibilidad para que el personal adquiera y mejore sus habilidades y conocimientos profesionales, ¿actualmente los administradores educativos brindan estos espacios de capacitación a su personal a cargo?

En la tabla 4 se visualizan los resultados obtenidos en el cuestionario aplicado a los docentes del CNLU en los cuales todas las variables fueron significativas pues el promedio de las respuestas positivas dadas a cada una de ellas es de $93 \%$ (33 docentes en promedio) muy superior al promedio de respuestas negativas que corresponde a $7 \%$ ( 2 docentes en promedio). 
TABLA 4

Resumen de las respuestas dadas por los docentes con respecto a las acciones de formacion continua que estimulen la adquisición de habilidades profesionales

\begin{tabular}{|c|c|c|c|c|}
\hline \multirow[b]{2}{*}{ Variables } & \multicolumn{2}{|c|}{$\begin{array}{l}\text { Respuestas negativas: } \\
\text {-Muy en desacuerdo } \\
\text {-En desacuerdo } \\
\text {-Ni de acuerdo ni en desacuerdo }\end{array}$} & \multicolumn{2}{|c|}{$\begin{array}{l}\text { Respuestas positivas: } \\
\text {-Muy de acuerdo } \\
\text {-De acuerdo }\end{array}$} \\
\hline & $\begin{array}{c}\text { Frecuencia } \\
\text { Absoluta }\end{array}$ & $\begin{array}{c}\text { Frecuencia } \\
\text { Relativa }\end{array}$ & $\begin{array}{c}\text { Frecuencia } \\
\text { Absoluta }\end{array}$ & $\begin{array}{c}\text { Frecuencia } \\
\text { Relativa }\end{array}$ \\
\hline $\begin{array}{l}\text { Pregunta 15: ¿Considera necesarias acciones de formación } \\
\text { continua que estimulen habilidades profesionales que propicien } \\
\text { una mejor atención a la comunidad del colegio nocturno? }\end{array}$ & 0 & $0 \%$ & 35 & $100 \%$ \\
\hline $\begin{array}{l}\text { Pregunta 16: ¿Considera necesarias acciones de formación } \\
\text { continua que estimulen habilidades profesionales que propicien } \\
\text { mejores estrategias de enseñanza-aprendizaje dirigidas a los } \\
\text { estudiantes adultos? }\end{array}$ & 2 & $6 \%$ & 33 & $94 \%$ \\
\hline $\begin{array}{l}\text { Pregunta 17: ¿Considera necesarias acciones de formación } \\
\text { continua que estimulen habilidades profesionales que propicien } \\
\text { un mejor entendimiento de los lineamientos y razón de ser de los } \\
\text { colegios nocturnos? }\end{array}$ & 3 & $9 \%$ & 32 & $91 \%$ \\
\hline $\begin{array}{l}\text { Pregunta 18: ¿Considera necesarias acciones de formación } \\
\text { continua que estimulen habilidades profesionales que permitan } \\
\text { efectuar un diagnóstico socioeducativo del estudiante adulto? }\end{array}$ & 4 & $11 \%$ & 31 & $89 \%$ \\
\hline $\begin{array}{l}\text { Pregunta 19: ¿Considera usted necesarias acciones de formación } \\
\text { continua que estimulen habilidades profesionales que propicien } \\
\text { un conocimiento del contexto donde está inmersa la población } \\
\text { estudiantil adulta? }\end{array}$ & 3 & $9 \%$ & 32 & $91 \%$ \\
\hline Promedio & 2,4 & $7 \%$ & 32,6 & $93 \%$ \\
\hline
\end{tabular}

Fuente: Víquez (2017, p. 168)

Por tanto, según los resultados anteriores existe una gran necesidad de formación continua que debe ser solventada mediante acciones propias de la administración del centro educativo.

De acuerdo con los resultados obtenidos es recomendable que para este tipo de instituciones educativas se establezcan políticas internas, las cuales propicien el conocimiento de las características propias de los colegios nocturnos.

Los resultados cualitativos respaldan los hallazgos a nivel cuantitativo, por ejemplo, el director menciona:

[...] como vas a educar al que no conoces. Eso es muy sencillo, a la comunidad de Tres Ríos hay que conocerla, de alguna manera debemos traer el conocimiento de la comunidad [...] (Vargas, 2017).

Lo anterior, haciendo alusión a la importancia de conocer el contexto donde está ubicada la institución educativa, y de cierta manera también de los mismos estudiantes, ya que un reflejo de la comunidad son los mismos discentes. 
De lo expuesto en este apartado, se destaca la necesidad de que el administrador educativo adopte los principios de la gestión educativa estratégica y los adecue a la realidad institucional, principalmente los procesos de planificación, con el fin de ajustarlos a las características propias de este tipo de instituciones y así solventar las necesidades de formación detectadas en el personal.

\section{Taller de formación continua que favorezca la generación de acciones educativas hacia el personal}

Como respuesta al diagnóstico efectuado se propuso el desarrollo de un taller que diera solución al problema planteado consistente en elaborar acciones de formación continua que desde el modelo de gestión educativa estratégica promovieran el desarrollo del personal mediante la adquisición de habilidades profesionales.

¿Por qué se definió implementar un taller sobre los principios de la andragogía en el CNLU?, el taller es una alternativa que debe generarse desde la propia administración de la institución, ya que es una manera directa y eficiente de atender la necesidad de capacitación del personal, en la tabla 5 observamos la opinión de los docentes al respecto.

TABLA 5

Resumen de las respuestas dadas por los docentes con respecto al taller de formación continua que favorezca la generación de acciones educativas hacia el personal

\begin{tabular}{|c|c|c|c|c|}
\hline \multirow[b]{2}{*}{ Variables } & \multicolumn{2}{|c|}{$\begin{array}{l}\text { Respuestas negativas: } \\
\text {-Muy en desacuerdo } \\
\text {-En desacuerdo } \\
\text {-Ni de acuerdo ni en desacuerdo }\end{array}$} & \multicolumn{2}{|c|}{$\begin{array}{l}\text { Respuestas positivas: } \\
\text {-Muy de acuerdo } \\
\text {-De acuerdo }\end{array}$} \\
\hline & $\begin{array}{c}\text { Frecuencia } \\
\text { absoluta }\end{array}$ & $\begin{array}{c}\text { Frecuencia } \\
\text { relativa }\end{array}$ & $\begin{array}{c}\text { Frecuencia } \\
\text { absoluta }\end{array}$ & $\begin{array}{c}\text { Frecuencia } \\
\text { relativa }\end{array}$ \\
\hline $\begin{array}{l}\text { Pregunta 20: ¿Considera necesario el desarrollo de un módulo } \\
\text { de formación continua en el colegio sobre los principios de la } \\
\text { educación andragógica y la sensibilización del docente hacia los } \\
\text { estudiantes adultos? }\end{array}$ & 1 & $3 \%$ & 34 & $97 \%$ \\
\hline $\begin{array}{l}\text { Pregunta 21: ¿Considera necesario el desarrollo de un módulo de } \\
\text { formación continua en el colegio sobre el manejo y control del } \\
\text { aula con estudiantes adultos? }\end{array}$ & 5 & $14 \%$ & 30 & $86 \%$ \\
\hline $\begin{array}{l}\text { Pregunta 22: ¿Considera necesario el desarrollo de un módulo de } \\
\text { formación continua sobre estrategias de enseñanza y aprendiza- } \\
\text { je con estudiantes adultos? }\end{array}$ & 2 & $6 \%$ & 33 & $94 \%$ \\
\hline $\begin{array}{l}\text { Pregunta 23: ¿Considera necesario el desarrollo de un módulo de } \\
\text { formación continua en el colegio sobre liderazgo y motivación al } \\
\text { estudiante adulto? }\end{array}$ & 1 & $3 \%$ & 34 & $97 \%$ \\
\hline $\begin{array}{l}\text { Pregunta 24: ¿Considera necesario el desarrollo de un módulo } \\
\text { de formación continua en el colegio sobre los lineamientos de la } \\
\text { evaluación andragógica? }\end{array}$ & 4 & $12 \%$ & 31 & $88 \%$ \\
\hline Promedio & 2,6 & $7,6 \%$ & 32,4 & $92,4 \%$ \\
\hline
\end{tabular}

Fuente: Víquez (2017, p. 173) 
Los docentes fueron contundentes con respecto a la necesidad de formación profesional en la temática de andragogía, lo cual se evidencia al observar en la tabla 5 la solicitud de capacitación en temas como los principios de la andragogía, el manejo en el aula de los discentes, estrategias de enseñanza-aprendizaje y motivación de los estudiantes adultos.

Para todas las variables del estudio la incidencia fue significativa, un 92,4\% (32 docentes en promedio) se manifestó a favor, muy superior al promedio de respuestas negativas que corresponde a un 7,6\% (3 docentes en promedio).

Es importante insistir en que los estudiantes adultos poseen una serie de características muy particulares por ser una población estudiantil diferenciada, manifiestan una motivación personal por estudiar y son muy comprometidos con la institución educativa.

El discente adulto presenta situaciones personales especiales como tener que distribuir su tiempo entre la familia, el trabajo y estudio; los profesionales que atienden estos centros educativos deben capacitarse, para poder conocer, abordar y desarrollar el proceso educativo de la mejor manera.

\section{CONCLUSIONES}

La investigación realizada permitió concluir que existe una gran necesidad de capacitación en la temática de andragogía, los resultados encontrados evidencian las deficiencias profesionales existentes en el personal con respecto al correcto abordaje de los discentes adultos en las aulas y la correcta gestión institucional que debe desarrollarse en este tipo de centros educativos.

Un aporte muy importante de la investigación es demostrar que la atención que deben recibir los estudiantes en un colegio académico nocturno es completamente distinta a la que se imparte en un colegio diurno, en el cual se atienden adolescentes y niños, se demostró que lo más apropiado según la modalidad y el tipo de población estudiantil que se atiende es incorporar los principios establecidos por la andragogía base de la educación de adultos, en lugar de enfatizar en los aspectos pedagógicos como actualmente está establecido.

Un aspecto que debe llamar a la reflexión por parte de las universidades, basado en los resultados obtenidos, es con respecto a la formación profesional del personal docente, administrativo y técnico docente ya que en temáticas asociadas a la educación de adultos es escasa o nula la preparación recibida.

Ligado a lo anterior, se puede expresar que se están formando docentes y administradores educativos solamente preparados en pedagogía, dejando de lado la gran posibilidad de que docentes y administradores trabajen en modalidades educativas para adultos, por lo tanto, se apela a una preparación integral del personal desde su base profesional, tomando en cuenta las variables expuestas anteriormente, y así un profesional en educación sea capaz de adaptarse de manera exitosa en cualquiera que sea la modalidad de desempeño laboral.

Otro aporte muy interesante del estudio fue que los resultados señalaron que un director de un colegio académico nocturno debe poseer una serie de características personales y profesionales diferenciadas, que le permitan gestionar de manera exitosa este tipo de instituciones educativas.

Así, debe propiciar el trabajo en equipo, debe ser participe en los procesos de enseñanza y aprendizaje, debe involucrarse e involucrar a la comunidad con el centro educativo y tener una gran disposición para resolver los conflictos que a nivel interno se puedan presentar en la institución.

Muy relacionado con lo anterior, a partir de los resultados obtenidos se concluye que un director de un colegio nocturno debe ser una persona de trato amable tanto con estudiantes y personal a su cargo, 
debe ser muy humano, con una mística para relacionarse que lo diferencie, ya que debe ser sensible ante las diferentes situaciones que pueden presentar los estudiantes y los mismos docentes, sin dejar de lado su papel como administrador educativo.

Por otra parte, un director que se desempeñe profesionalmente en educación de adultos debe ser formado en administración educativa y poseer estudios a nivel andragógico, con el fin de manejar integralmente todas las posibles situaciones laborales, así como una persona con vasta experiencia en puestos de dirección y conocimientos en todos los procesos asociados a la gestión educativa estratégica.

De acuerdo con los resultados obtenidos se concluye que son necesarias acciones inmediatas por parte de la administración del centro educativo que propicien la adquisición de habilidades profesionales en el personal, con lo cual se pretende mejorar el servicio educativo ofrecido.

Se recalca nuevamente que no es lo mismo administrar una institución educativa diurna que una nocturna: la población estudiantil es diferente, se comparten instalaciones, los docentes vienen de trabajar una jornada diurna, todos aspectos que deben ser tomados en consideración.

Por último y partiendo de la necesidad de capacitación manifestada, se generó una propuesta de taller de capacitación que, a nivel interno, la dirección de este tipo de instituciones educativas podría desarrollar para solventar las deficiencias encontradas.

Un taller de capacitación en la temática de andragogía es un instrumento importante para mejorar las habilidades profesionales del personal en las áreas específicas que requieran ser fortalecidas en instituciones educativas para adultos.

Lo anterior es factible si existe voluntad por parte de la administración en primera instancia y disposición del personal para generar los espacios requeridos de tiempo en los cuales se pueda desarrollar.

A partir de los resultados obtenidos, es urgente que a nivel institucional se brinden los espacios para el desarrollo de los talleres propuestos, uno de los aspectos que justifica en mayor medida su implementación es la población estudiantil adulta, a la cual profesionalmente el personal no está capacitado para atender.

\section{REFERENCIAS}

Camargo, M. (Dir.). (2007). La formación de profesores en Colombia: necesidades y perspectivas. Bogotá: Universidad de la Sabana.

Chiavenato, I. (2009). Gestión del talento humano. México: McGrawHill.

Comisión Nacional de Rectores. (2015). Informe V. Estado de la Educación 2015. San José: CONARE.

Duarte, I. (8 de marzo de 2017). Comunicación personal sobre andragogía, necesidades de capacitación y gestión educativa estratégica. Víquez, E. Entrevistador. La Unión, Cartago. Costa Rica.

Freire, P. (1972). La educación como práctica de la libertad. México: Siglo Veintiuno.

Galiano, P. (2006). Informe 2: Observatorio Empleo Agrario Andaluz. Sevilla: Sevilla Siglo XXI.

Hernández, R., Fernández, C. \& Baptista, P. (2010). Metodología de la investigación. México: McGraw Hill.

Hurtado, J. (2010). Metodología de la investigación: guía para una comprensión holística de la Ciencia. Caracas: Quirón Ediciones.

Imbernón, F. (2007b). La formación y el desarrollo profesional del profesorado. Hacia una nueva cultura profesional. Barcelona: Grao. 
Knowles, M., Holton, E. \& Swanson, R. (2005). The Adult Learner: The Definitive Classic in Adult Education and Human Resource Development. Houston: Gulf Publishing Company.

Ministerio de Educación de la Nación. (2015). Gestión educativa estratégica. Módulo 2 de 10. Buenos Aires: Instituto de Planeamiento de la Educación.

Navío, A. (2005). Propuestas conceptuales en torno a la competencia profesional. Revista de Educación. (337), 213-234.

Sánchez, A. (22 de agosto 2016). Comunicación personal sobre la temática de gestión educativa. (Viquez, E. Entrevistador). La Unión. Cartago. Costa Rica.

Sanz. F., \& Lancho. J. (2012). Fundamentos de la educación de personas adultas, módulos 1, 2, 3, 4. Madrid: Ministerio de Educación, Instituto de Tecnologías Educativas.

UNESCO. (1985). Cuarta Conferencia Internacional sobre la Educación de Adultos. Paris: UNESCO.

Vargas, J. (13 de marzo de 2017). Comunicación personal sobre andragogía, necesidades de capacitación y gestión Educativa estratégica. Víquez, E. Entrevistador. La Unión, Cartago. Costa Rica

Víquez, E. (2017). Propuesta de capacitación que desde la gestión educativa estratégica promueva la formación del personal docente y técnico docente mediante la adquisición de habilidades profesionales que coadyuven al fortalecimiento de la dimensión andragógica-curricular en el Colegio Nocturno La Unión de Tres Ríos. San José. Costa Rica. Universidad Estatal a Distancia.

Yturralde, E. (2016). Educación andragógica, pedagogía y andragogía. Recuperado de: http://www.yturralde.com/andragogia.htm 